\title{
Rol del gabinete logopédico en la inclusión educativa de jóvenes universitarios con diagnóstico de tartamudez
}

\section{Role of the speech therapy cabinet in the educational inclusion of young university students with a diagnosis of stuttering}

\author{
Yunia García \\ Universidad Central "Marta Abreu" de Las Villas. Santa Clara. Villa Clara. Cuba \\ ORCID: https://orcid.org/0000-0002-9781-5091 \\ Tania Hernández \\ Universidad Central "Marta Abreu" de Las Villas. Santa Clara. Villa Clara. Cuba \\ ORCID: https://orcid.org/0000-0002-0409-9269 \\ Tamara B. Hernández \\ Universidad Central "Marta Abreu" de Las Villas. Santa Clara. Villa Clara. Cuba \\ ORCID: https://orcid.org/0000-0002-2643-673X

\section{Pablo A. Martínez} \\ Universidad Central "Marta Abreu” de Las Villas. Santa Clara. Villa Clara. Cuba \\ ORCID: https://orcid.org/0000-0001-5529-8199
}

*Correspondence

Email: ygmanso@uclv.cu
Cite as:

García, Y., Hernández, T., Hernández, T. B \& Martínez, P. A. (2020). Rol del gabinete logopédico en la inclusión educativa de jóvenes universitarios con diagnóstico de tartamudez. Propósitos y Representaciones, 8 (SPE3), e736. Doi: http://dx.doi.org/10.20511/pyr2020.v8nSPE3.736 


\section{Resumen}

La Educación Superior en Cuba se encuentra en un particular proceso de transformación; en efecto, el desarrollo de la sociedad del conocimiento plantea nuevas exigencias a las universidades. Una de ellas es precisamente la inclusión educativa de los jóvenes universitarios, para el logro de las competencias profesionales necesarias en su desempeño. Particularmente aquellos que poseen diagnóstico de tartamudez y que estudian carreras pedagógicas, necesitan de una especial atención por los especialistas del lenguaje (logopeda) de manera que puedan alcanzar la seguridad e independencia que exige el ejercicio de la profesión. El gabinete logopédico es un espacio por excelencia para favorecer la inclusión educativa de los jóvenes universitarios con diagnóstico de tartamudez, sin embargo, existe un limitado nivel de conocimientos por parte de profesores, estudiantes y directivos acerca del rol que desempeña en este sentido. Desde esta perspectiva de análisis el objetivo del artículo está centrado en: Valorar la labor que se realiza desde el gabinete logopédico de la Universidad Central "Marta Abreu" de Las Villas (provincia Villa Clara, Cuba), para favorecer la inclusión educativa de una joven con diagnóstico de tartamudez. En el logro este propósito, se desarrolló una investigación de tipo exploratoria, a través de un estudio de caso, que constituyó la fase inicial de un estudio posterior de mayor profundidad. Como resultado se obtuvo el diagnóstico del estado real de la labor que se realiza desde el gabinete logopédico, en relación a la inclusión educativa de una joven con diagnóstico de tartamudez.

Palabras clave: Inclusión Educativa; Gabinete Logopédico; Tartamudez.

\section{Summary}

Higher Education in Cuba is a particular transformational process; indeed, the development of knowledge in the society requires new demands in the universities. One of this demand is precisely the educational inclusion of young university students, for the achievement of the necessary professional skills in their performances. Particularly, those who have a diagnosis on stuttering and the ones who are studying pedagogical careers, need special attention by a language specialist (speech therapist) so that they can achieve their independence required by the exercise of the profession from a quality education. The speech therapy cabinet office is an excellent place to favor the educational inclusion of young university students with a diagnosis of stuttering, however, there is a limited level of knowledge by teachers, students and managers about the role they play in this regard. This article is aimed at evaluating the work that is done from the speech therapy cabinet of the Central University "Marta Abreu" de Las Villas (Villa Clara province, Cuba), to favor the educational inclusion of a young woman with Stuttering diagnosis. In order to achieve this purpose, an exploratory research was implemented through a case study, which constituted the initial phase of a further study. As a result, the diagnosis of the real state of the work that is carried out from the speech therapy cabinet was obtained, in relation to the educational inclusion of a young woman with a diagnosis of stuttering.

Keywords: Educational Inclusion; Speech Therapy Cabinet; Stuttering.

\section{Introducción}

Desde finales del siglo XX e inicios del XXl, los profundos cambios en la realidad mundial contemporánea, han condicionado un perfeccionamiento continuo de los diferentes sistemas educacionales. En la actualidad existen marcadas diferencias entre las condiciones del mundo moderno y el de hace apenas unas décadas, dadas por el complejo proceso de globalización que vive la humanidad.

El reto es cambiar y desarrollar nuevas políticas y estrategias para dar respuesta efectiva a las problemáticas que en ella se desarrollan. Desde este complejo escenario emerge la polémica acerca de: la integración educativa, la atención a la diversidad y la inclusión educativa. 
Estos temas han provocado debates y controversias, con el propósito de hacer frente a los altos índices de exclusión, discriminación y desigualdades educativas, presentes en la mayoría de los sistemas educativos del mundo. Desde esta lógica, una escuela inclusiva ofrece a todos sus alumnos las oportunidades, y los apoyos necesarios, de orden curricular, personal y material, entre otros, para su progreso académico y personal.

Cuba no ha estado ajena a estos cambios; se realizan esfuerzos significativos en cada uno de los niveles educacionales (preescolar, primaria, secundaria básica, preuniversitario, universidad), para llevar una educación con calidad a todos los niños, adolescentes y jóvenes.

En medio de la cotidianidad de la escuela, resulta imprescindible formarlos en los mejores valores de su época, en los más avanzados adelantos de la ciencia, con una formación integral que les permita interactuaren la sociedad con seguridad e independencia.

En el camino hacia el cumplimiento de la Agenda 2030 y de los Objetivos de Desarrollo Sostenible formulados por Naciones Unidas (ONU, 2015), la educación cubana ha desarrollado directrices nacionales de trabajo, e importantes proyectos educativos, con el propósito de avanzar en el objetivo 4, que plantea la necesidad de brindar una educación de calidad, y en pos de alcanzar la meta referida, al aumento del número de jóvenes que tienen las competencias necesarias, en particular las profesionales, para acceder al empleo y desempeñarse en el eslabón de base de su profesión.

Educación para todos durante toda la vida, es el objetivo supremo asumido por la UNESCO para caracterizar la nueva cualidad que debe estar presente en la educación en la época actual. Esta tesis es igualmente válida, cuando se profundiza en el verdadero papel correspondiente a las universidades de hoy. (Horruitiner, 2009, p.7)

En este empeño, la inclusión educativa de los jóvenes que ingresan a la universidad, constituye una prioridad. El carácter eminentemente humanista de las universidades cubanas hace que la "visión de la formación inicial rebase lo instructivo, lo meramente cognitivo y centre su atención fundamental en el hombre, en el desarrollo pleno de su personalidad (...)" (Horruitiner, 2009, p.16).

A pesar de la importancia que se le concede a la inclusión educativa desde este nivel educacional; el proceso para docentes y directivos, se presenta aún, lleno de incertidumbres y dudas por la magnitud y diversidad de su manifestación en la práctica. Todavía es un reto en la actualidad, la inclusión educativa de los jóvenes con tartamudez, que ingresan a la universidad para estudiar carreras pedagógicas y ejercer su vida profesional como maestros.

En este sentido autores como Castejón, González, Núñez \& González (2008) refieren que:

Los alumnos tartamudos demandan a la escuela inclusiva una transformación en dos aspectos: un estilo de la relación maestro-alumno que preste más atención a variables afectivo-personal, una mayor atención a la integridad de la persona evitando la centración de la educación en los aspectos estrictamente académicos. (p. 90)

Aunque cada vez hay más información científica sobre la atención logopédica a la población con tartamudez en el marco escolar, la complejidad en la corrección y/o compensación de este trastorno, su naturaleza multicausal y las carencias en la atención pedagógica, inciden en su durabilidad y agravamiento.

Gallego (2013) afirma que: "Este trastorno, que condiciona la funcionalidad de la comunicación en su conjunto, es de naturaleza multicausal, tenaz variabilidad, pronóstico incierto y tiene o puede tener implicaciones en diferentes ámbitos del desarrollo (...)" (p. 95). 
No es un hecho aislado encontrar a jóvenes, cuya vida escolar transcurrió bajo la incidencia de un especialista del lenguaje (logopeda), y que mantienen rasgos distintivos del trastorno, o este, en toda su expresión. ¿Sería justo para ellos impedirles u obstaculizarles el estudio de la profesión que han escogido?

A pesar de los avances de la ciencia y la velocidad de estos, la tartamudez, continúa siendo un enigma tanto para los que la sufren, como para aquellos especialistas que se han empeñado en buscar un tratamiento para la misma.

Beain (2015) al referirse a los jóvenes con tartamudez plantea:

(...) el joven que presenta tartamudez, ya ha probado diversos tratamientos, en los que ha tenido escaso éxito(...)tiene un sufrimiento oculto que, raras veces ha compartido con alguien y ya lleva vividas una serie de experiencias traumáticas con respecto a su habla que le han ido perfilando una determinada personalidad. (p. 13)

Por su parte Garaigordobil \& Pérez (2007) refieren que:

Es importante denotar que la alteración de la fluidez interfiere en el rendimiento académico, laboral o la comunicación social, y su intensidad varía en función de las situaciones, siendo a menudo más grave cuando se produce una presión especial para comunicar. (p.35)

En países como Estados Unidos de América y Australia, se han desarrollado investigaciones acerca de los efectos sociales que presentan los adultos jóvenes con tartamudez, en donde se evidencian algunas de las limitaciones en su participación.

Se reporta desde dichos estudios que, los adultos jóvenes que tartamudean no pueden identificarse a sí mismos como comunicadores eficaces, y mantienen limitaciones en los comportamientos necesarios para fomentar relaciones sociales entre pares (Blood \& Blood, 2004).

Los hallazgos de estas investigaciones, fortalecen la idea de continuar en la búsqueda de soluciones para fortalecer la atención logopédica a jóvenes universitarios con tartamudez.

Si han decidido formarse como maestros y ejercer una profesión, donde el uso de la palabra es vital para el aprendizaje y la formación integral de sus alumnos, es ineludible que reciban el acompañamiento y el apoyo de los profesores y especialistas de Logopedia de la universidad.

"Para el grupo de alumnos que tartamudea, la opción inclusiva representa la esperanza de que la escuela se transforme y desarrolle de tal manera que se eliminen las barreras que impiden su participación y hacen que se sientan excluidos" (Castejón et al., 2008, (p.282).

Autores como Ygual \& Cervera (1999), Gallego (1999), realizan una aproximación al marco actual de intervención logopédica en los trastornos del lenguaje y del habla. De igual manera lo hacen, Figueredo \& López (1984), (1986), Pérez (2002), Morales (2004), Monfort (2005), Chernousova (2008), Noguera (2008), Fernández (2008), Amaguaya (2013), Moreno (2017) y Arguelles (2019).

Estos autores han profundizado en las diferentes alteraciones del lenguaje y la comunicación, aportando sistemas de procedimientos, métodos y la concepción de atención a entidades logopédicas particulares; no obstante, los resultados dejan evidente la carencia del tratamiento teórico y metodológico a la concepción general de la atención logopédica desde el campo pedagógico, con una visión global que conciba la integración de este especialista al proceso docente-educativo y del resto de los agentes que intervienen en el proceso de atención logopédica. 
Resultan importantes para la investigación desarrollada, los estudios realizados por Bonilla, Bimilla \& Mosquera (2014) relacionados con las percepciones de las habilidades comunicativas, sociales cognitivas, psicoemocionales y psicomotrices de un grupo de jóvenes con tartamudez, así como los de Tovar (2016), en la que se destaca una conceptualización sobre la tartamudez y su implicación en la vida cotidiana de niños, adolescentes y adultos.

En coherencia con estos elementos, autores como Castejón\& España (2004), han publicado diversos trabajos en los que:

(...) apuestan por un modelo inclusivo logopédico en el que intervención esté enmarcada dentro del contexto social, escolar y familiar del sujeto. Se defiende la necesidad de la logopedia adaptarse a la realidad escolar contando con la colaboración del resto del profesorado, promoviendo una acción directa dentro del aula ordinaria. (p.41)

En un centro educativo con una orientación inclusiva, el equipo educativo, y no únicamente los maestros individualmente, encuentra en la atención a la diversidad un medio fundamental para mejorar la calidad educativa (Booth \& Ainscow,2000; Echeita \& Sandoval, 2002; Echeita, 2006).

Lo que distingue a un contexto inclusivo es la capacidad de respuesta hacia las necesidades educativas de los estudiantes. En un modelo inclusivo de universidad, las dificultades de los alumnos tartamudos adquieren relevancia, en tanto que constituyen una manifestación de la diversidad.

Desde la Universidad Central "Marta Abreu" de Las Villas, una alternativa de trabajo en este sentido, lo constituye el gabinete logopédico. Un espacio donde se le brinda especial atención a niños, adolescentes y jóvenes con trastornos del lenguaje y la comunicación, a partir del potencial científico del departamento docente de Educación Especial y los alumnos ayudantes, con particular énfasis en estudiantes universitarios.

En Cuba se reconocen como antecedentes de valor relacionados con la creación de gabinetes logopédicos desde contextos universitarios, el laboratorio logopédico de la Universidad "Hermanos Saíz Montes de Oca" de la provincia Pinar del Río (2012) y el gabinete de orientación logopédica que se desarrolla en la Universidad Pedagógica Juan Marinello Vidaurreta de la provincia de Matanzas, y los estudios realizados por Delgado, Navarro, Vigoa, Castro \& Martínez (2016), donde abordan al gabinete de orientación logopédica como espacio formativo y de interacción con la comunidad.

Por otra parte, se destacan Valdés, Fernández, Perojo (2017) quienes coinciden en la relevancia del laboratorio logopédico en la formación inicial y permanente en la universidad, así como Hernández, Hernández \& Rodríguez (2019), que refieren la importancia del gabinete logopédico desde el contexto universitario. ), y que uno de los propósitos fundamentales de este espacio, es ofrecer atención logopédica a jóvenes que ingresan a la universidad con trastornos del lenguaje y la comunicación, por la importancia que poseen no solo para su desarrollo profesional, sino también por el perfil del egresado de aquellos estudiantes que ingresan a carreras pedagógicas.

Aunque las prácticas inclusivas, y la correspondiente atención logopédica de jóvenes universitarios con diagnóstico de tartamudez desde el gabinete logopédico, se organiza a partir de normas y presupuestos teórico-prácticos que rigen este proceso en cualquier nivel educacional; el hecho de ser desarrolladas en el contexto universitario, con jóvenes que estudian carreras pedagógicas y que presentan este diagnóstico, le imprime, sin dudas, rasgos distintivos al proceso.

Es imprescindible considerar sus mecanismos de afrontamiento ante el trastorno; el grupo estudiantil donde se encuentran insertados, el comportamiento de sus profesores para cumplir las 
exigencias del proceso de formación inicial de un estudiante universitario, sin dejar de considerar las particularidades que manifiesta al poseer un diagnóstico de tartamudez.

El gabinete logopédico, es el espacio por excelencia desde donde se organizan la multiplicidad de influencias educativas que favorecerán la corrección y/o compensación del trastorno y la inclusión educativa de estos.

Su formación como maestros con la seguridad e independencia que exige el ejercicio de la profesión, tendrá como apoyo la labor de los especialistas en Logopedia, al realizar su labor terapéutica, de orientación y seguimiento; sin embargo, existe un limitado nivel de conocimientos por parte de profesores, estudiantes y directivos acerca del rol que se le otorga al gabinete logopédico en este empeño.

El presente artículo, resultado de una investigación científica, tiene como propósito valorar la labor que se realiza desde el gabinete logopédico de la Universidad Central "Marta Abreu" de Las Villas para favorecer la inclusión educativa de una joven con diagnóstico de tartamudez.

\section{Método}

El estudio que se presenta asume un enfoque cualitativo en su variante investigación acción colaborativa para dar tratamiento a las evidencias y medir el alcance de los resultados. En el análisis que se realiza se tiene en cuenta la subjetividad de los participantes en la práctica contextualizada en aras de aportar mayor objetividad al proceso.

Se trata de una investigación de tipo exploratoria, que constituyó la fase inicial de un estudio posterior de mayor profundidad.

La investigación se desarrolló desde el gabinete logopédico de la Universidad Central "Marta Abreu" de Las Villas (provincia Villa Clara, Cuba), lugar donde se atienden niños, adolescentes y jóvenes con trastornos del lenguaje y la comunicación, a partir del potencial científico del departamento docente de Educación Especial y los alumnos ayudantes. Dentro de esta diversidad se distingue la necesaria atención que requieren los estudiantes universitarios con diagnóstico de tartamudez para favorecer su inclusión educativa.

Se realizó un estudio de caso a una estudiante con diagnóstico de tartamudez, que cursa el segundo año de la carrera Licenciatura en Educación. Pedagogía-Psicología. Se incluyeron en el estudio tres docentes del departamento de Educación Especial, que realizan acciones desde el gabinete logopédico; dos alumnos ayudantes, cinco profesores que imparten docencia al caso seleccionado y 10 estudiantes del grupo.

Se aplicó la entrevista semiestructurada a los profesores y alumnos ayudantes que trabajan desde el gabinete logopédico con el caso seleccionado, así como a la estudiante con tartamudez, para constatar las particularidades del trabajo realizado desde este espacio en función de favorecer la inclusión educativa de la estudiante.

Otro método empleado fue la observación participante a diferentes actividades políticas, culturales, comunitarias, con el objetivo de constatar las características del trastorno que manifiesta la estudiante seleccionada, sus mecanismos de afrontamiento, y la dinámica dentro del espacio grupal donde se encuentra incluida.

Este método proporcionó información acerca de los beneficios de la atención que se ofrece al caso con tartamudez objeto de estudio desde el gabinete logopédico.

También se aplicó una encuesta a los estudiantes del grupo de segundo año y a profesores que trabajan con el caso, para constatar la importancia del gabinete logopédico en la inclusión educativa de la estudiante seleccionada. 
Se utilizó, además, el análisis de documentos, y se verificó la documentación que rige el funcionamiento del gabinete logopédico de la universidad central "Marta Abreu" de Las Villas.

Se efectuó la revisión del plan de trabajo metodológico del departamento al que corresponde la carrera Licenciatura en Educación. Pedagogía-Psicología, y al proyecto estudiantil del grupo donde se encuentra incluida la estudiante con diagnóstico de tartamudez, con el fin de constatar las acciones que se realizan para favorecer su inclusión educativa y conocer elementos relacionados con la historia de vida de la estudiante.

\section{Resultados}

Para realizar la valoración de la labor que se realiza desde el gabinete logopédico de la Universidad Central "Marta Abreu" de Las Villas, en aras de favorecer la inclusión educativa de una joven con diagnóstico de tartamudez, fue de vital importancia, la experiencia de las autoras en el trabajo desde este espacio como profesionales cuyo rol se relaciona con la disciplina Logopedia.

La información obtenida durante el proceso investigativo fue analizada e interpretada mediante la triangulación de fuentes, lo que permitió obtener datos complementados desde diferentes fuentes.

En entrevista realizada a los tres docentes del departamento de Educación Especial y a dos alumnos ayudantes, que realizaron acciones desde el gabinete logopédico, se constató que en la universidad donde se realiza el estudio, cada año se reciben jóvenes que presentan trastornos de la comunicación, donde se desataca por su incidencia, la tartamudez.

Este trastorno posee una gran repercusión a nivel psicológico y en la personalidad, por lo que existe la necesidad de favorecer la inclusión educativa de los jóvenes que lo portan.

Los entrevistados aportaron como datos de interés:

- La no ejecución de una prueba de aptitud para los estudiantes que ingresan a carreras pedagógicas, que permita identificar la presencia de trastornos de la comunicación y el lenguaje, que pudieran limitar su desempeño profesional como docentes.

- Las limitaciones en la percepción de riesgo de los docentes de la Educación Superior en relación a las consecuencias de la tartamudez para la vida emocional y la formación inicial de los estudiantes que la presentan.

- El limitado nivel de conocimientos por parte de profesores, estudiantes y directivos acerca del rol que se le concede al gabinete logopédico en la inclusión educativa de estudiantes con tartamudez.

La aplicación de la entrevista, reveló que el $100 \%$ coincide en que el gabinete logopédico ofrece grandes posibilidades para la realización de una terapia correctiva/compensatoria a los jóvenes con tartamudez y para favorecer la inclusión educativa de los mismos.

Dos de los profesores de mayor experiencia aportaron como datos de valor, que en el inicio de cada curso escolar se realiza un estudio exploratorio, por parte de los docentes que brindan atención desde el gabinete logopédico, para realizar la detección de los jóvenes universitarios que han ingresado a la Educación Superior con trastornos en el lenguaje, y particularmente con tartamudez.

Derivada de esta acción, según los entrevistados, se concibieron acciones para la atención individual y grupal de la estudiante seleccionada, se ofrecieron orientaciones a su familia, se 
desarrollaron dinámicas de grupo para favorecer su inclusión educativa y se capacitó a los docentes de diferentes asignaturas que interactuaban con ella.

Según la opinión del $100 \%$ de los alumnos ayudantes, esta labor incidió de forma muy positiva en la estudiante con diagnóstico de tartamudez pues no solo favoreció la corrección del trastorno, sino también sus relaciones interpersonales, su seguridad en clases y su autoestima.

La estudiante con diagnóstico de tartamudez expresó durante la entrevista que antes de recibir atención desde el gabinete logopédico, se sentía en ocasiones excluida, poco comprendida y con la vergüenza de la burla por parte de algunos de sus compañeros.

Refirió, además, que en la actualidad siente que sus profesores toman en consideración sus posibilidades durante la concepción de las diferentes clases y actividades como estudiante universitaria, brindan más posibilidades para su participación, y que por tal razón sus resultados también han mejorado.

Durante la observación participante se pudo apreciar que la estudiante, presenta una tartamudez tonoclónica, con la presencia de sonidos fóbicos y contracciones espasmoideas que limitan su comunicación; dificultades en la coordinación fono-respiratoria y limitaciones en sus relaciones interpersonales.

A partir de las sesiones de terapia recibidas desde el gabinete logopédico, fue posible observar el desarrollado de habilidades que le han permitido una mejor comunicación, a partir de lograr la identificación de los sonidos fóbicos, y la sustitución de palabras donde estos se encuentran, por otras que son sinónimos.

Se comunica de forma más fluida, con menor cantidad de retrocesos, repeticiones. Solo se mantienen ligeras contracciones espasmoideas y los movimientos concomitantes de los ojos. Se implica en las actividades que se realizan y ha elevado la participación en clases.

Es posible apreciar que sus compañeros han aprendido a escucharla sin mostrar signos de desesperación, la incluyen en mayor medida durante las tareas docentes, actividades recreativas, deportivas y culturales. Se siente mejor comprendida y apoyada dentro de la institución.

Se observa su satisfacción con la atención logopédica recibida desde el gabinete logopédico y que esta ha favorecido en gran medida su inclusión educativa, lo que queda evidenciado en los avances obtenidos.

Mediante la encuesta realizada a los cincoprofesores que imparten clases de diferentes asignaturas a la estudiante seleccionada (33,3\%), se pudo constatar que se encuentran mejor preparados para contribuir a su inclusión educativa, a partir de la influencia que se ha ejercido sobre ellos desde el gabinete logopédico.

En el análisis de la encuesta se observa coincidencia, en que la estudiante al inicio de su ingreso a la universidad, se mostraba insegura y ansiosa ante las actividades de expresión oral, en que eran frecuentes las conductas de evitación al leer en voz alta y su negativa para responder preguntas.

En sus respuestas fue evidente que en la actualidad ha ganado en autocontrol, se esfuerza por hacerse entender. Se le ofrece una atención diferenciada durante sus respuestas en seminarios, clases prácticas orientadas, y otras actividades curriculares.

Refieren además que, cuando se presentan las contracciones espasmoideas y los movimientos concomitantes, actúan con normalidad, y aplican las recomendaciones que se le han 
sugerido desde el gabinete logopédico. Expresan que los compañeros de aula, también han avanzado en este sentido y la apoyan.

Reconocen la importancia del gabinete logopédico como espacio para la inclusión educativa de los jóvenes universitarios que ingresan a carreras pedagógicas con tartamudez, y se sienten mejor preparados para cumplir con su rol profesional.

Expresan que, para la evaluación de la estudiante, ponderan las formas escritas sobre las orales. No realizan correcciones, ni observaciones a la manera en la que se comunica, valorando más lo que dice que la forma en que lo dice.

Los 10 compañeros de estudio encuestados $(77,7 \%)$ reconocen la importancia del gabinete logopédico para la inclusión educativa de la estudiante seleccionada, pues se ha integrado más al grupo, se muestra más dispuesta para participar en clases y en las demás actividades convocadas, cuatro de ellos $(40 \%)$ coinciden en plantear que presenta con menor frecuencia los espasmos y titubeos.

Como resultado del análisis de documentos se constató el incremento de acciones encaminadas a favorecer la inclusión educativa de la joven con tartamudez. De manera planificada y consciente se realizaron actividades que favorecieron su desarrollo integral desde el ámbito laboral, extensionista, académico e investigativo.

En los documentos analizados se apreció la realización de diversas actividades y acciones desde el gabinete logopédico, con el propósito de contribuir al proceso de inclusión educativa de la joven con tartamudez. Se destacan las acciones de capacitación a los docentes, la realización de actividades demostrativas sobre la dirección del proceso de enseñanza-aprendizaje con la inclusión de la joven tartamuda, las sesiones de orientación a su familia y las sesiones de terapia a la joven seleccionada para el estudio.

Desde el trabajo metodológico del departamento al que corresponde la carrera que estudia la joven seleccionada para el estudio (Licenciatura en Educación. Pedagogía-Psicología), se destaca la realización de actividades metodológicas de preparación con los profesores, la incorporación de acciones diferenciadas en la estrategia educativa de su año de estudio y en el proyecto estudiantil del grupo donde se encuentra incluida.

\section{Discusión}

El análisis integrador de los diferentes instrumentos aplicados en la investigación, permitió valorar la labor que se realiza desde el gabinete logopédico de la Universidad Central "Marta Abreu" de Las Villas para favorecer la inclusión educativa de una joven con diagnóstico de tartamudez.

El estudio de caso realizado con la estudiante seleccionada, favoreció la obtención de datos científicamente comprobables sobre el trabajo del gabinete logopédico en su función correctiva-compensatoria, de orientación y seguimiento, así como su influencia positiva hacia todos los factores implicados en la inclusión de la estudiante (grupo, profesores, familia).

La investigación reveló las insatisfacciones y las vivencias negativas que, en la etapa inicial del ingreso a la universidad, había tenido la estudiante; sus limitaciones en el establecimiento de relaciones con sus compañeros y las burlas de algunos de ellos.

En relación con este aspecto, Gallego (2016), apunta: "Estas alteraciones causan ansiedad al hablar y limitaciones en la comunicación efectiva, que interfiere de forma aislada o de forma combinada en la participación social (...)"(p.60).

Por su parte, Rodríguez (2002), en un estudio sobre la tartamudez desde la perspectiva de las personas tartamudas, destaca como estas atribuyen a los años escolares consecuencias nefastas 
para su futuro ajuste personal y social: "Fueron los peores años de mi vida. La escuela, por culpa de mi tartamudez, fue un sufrimiento"(p. 83).

Fue posible conocer, además, las consecuencias negativas de la tartamudez en el desempeño académico y en el desarrollo integral de la joven seleccionada; se coincide con Koplas \& Ross (2004), en el impacto negativo de la tartamudez en la relación interpersonal que los alumnos tartamudos mantienen con compañeros y profesores en el contexto escolar, y también, en el impacto de la misma en el rendimiento académico.

En este sentido Guitar (1998), apunta que:

(...) aunque los alumnos tartamudos suelen tener capacidades suficientes para tener un buen rendimiento, sus resultados se ven con frecuencia limitados por las dificultades de participación en el aula y por las limitaciones que la tartamudez conlleva en el ámbito escolar. (p.283)

Se comprobó que la orientación sistemática hacia los alumnos del grupo, la dirigida a los profesores y a la familia de la joven, repercutió en la mejor comprensión del trastorno, en la ayuda pedagógica, en sus relaciones sociales, en la creación de un clima de confianza y empatía entre los que interactuaban con ella en el contexto universitario; todo ello, evidencia de una mejor inclusión educativa.

Miras (1998), refiere que: "los alumnos y los profesores que interactúan (...) además de poner en juego sus capacidades y recursos cognitivos, experimentan sentimientos, deseos, intereses que denotan sus correspondientes capacidades afectivas y de equilibrio personal" (p.47).

Desde esta perspectiva se toman en consideración a los autores Both \& Ainscow (2000), al plantear que:

(...) en una propuesta de atención a la diversidad en el marco de la escuela inclusiva, surge una cuestión esencial relacionada con el cambio educativo: si las propuestas inclusivas enriquecen la calidad educativa de la escuela, habría que preguntarse qué dimensiones del proceso de enseñanza-aprendizaje son relevantes cuando se habla de las dificultades de los alumnos tartamudos y si el resto del alumnado puede beneficiarse de la atención a esos aspectos. Estas dos cuestiones garantizan la viabilidad del cambio educativo, pues, en un marco inclusivo, no se pretende trabajar sólo para un grupo de alumnos, sino para todo el alumnado y con el compromiso de generar un cambio social. (p.36)

Se coincide con Marchesi (2004) al enfatizar en que:

El profesor ha de ser consciente de que en las relaciones que se establecen en su aula se está creando un mundo de relaciones y de afectos. Y que él es un punto de referencia afectivo importante para cada uno de sus alumnos. Su manera de comunicarse, de organizar el trabajo en clase, de atender y evaluar a sus alumnos va a tener una indudable repercusión sobre ellos. (p.145)

Fue posible inferir que los jóvenes tartamudos encuentran en la universidad factores de riesgo que repercuten tanto en la tartamudez, como en su ajuste afectivo-personal, y que tienen importantes efectos en su desarrollo personal y en su integración social. Las barreras a las que se enfrentan en su vida universitaria son un reto para impulsar el cambio en los centros universitarios.

En un contexto inclusivo, las dificultades de los alumnos tartamudos deben atenderse de acuerdo con criterios educativos que permitan potenciar los factores de protección frente a los de riesgo. Cuando se habla de inclusión hay que centrarse en un grupo de alumnos en situación de riesgo de exclusión; descubrir las barreras que estos encuentran para el aprendizaje y la participación. Estas son un reto para impulsar valores y prácticas inclusivas que fomenten el desarrollo de los centros (Both \& Ainscow, 2000; Echeita, 2006).

El estudio exploratorio efectuado demostró que el gabinete logopédico juega un papel fundamental, en un marco colaborativo, para llegar a entender la tartamudez, no solo como trastorno, sino como fuente de dificultades educativas en la relación del joven con su entorno. 
Tal como señalan Both \& Ainscow (2000): "centrarse en las dificultades de ese colectivo de alumnos puede desviar la atención de las dificultades experimentadas por otros alumnos" (p. 9).

El establecimiento de un ambiente de participación y colaboración entre los profesores, los alumnos del grupo y los especialistas del lenguaje que laboran en el gabinete logopédico, permitió socializar las dificultades experimentadas como vivencias compartidas para construir un marco inclusivo más allá del aula, y del momento en que puntualmente maestro y alumno interactúan.

Como plantea Castejón et al. (2008): “debe promoverse en el equipo educativo un proceso inclusivo para crear un marco organizativo y un clima de colaboración que dé respuesta a las dificultades que surgen, en un momento dado, de la diversidad del alumnado". (p.293)

\section{Conclusiones}

Desde la acción del gabinete logopédico, se desarrolló un conocimiento más profundo sobre la tartamudez; se fortaleció la promoción de acciones correctivas y compensatorias ante el trastorno, ocurrió el fomento de actitudes favorables hacia la inclusión educativa de la estudiante con tartamudez, la búsqueda de procedimientos de participación oral que favorecieran la intervención de todo el alumnado, así como la atención logopédica individualizada y actividades conjuntas con el grupo.

La triangulación de los datos permitió realizar una valoración positiva de la labor que se realiza desde el gabinete logopédico de la Universidad Central "Marta Abreu" de Las Villas para favorecer la inclusión educativa de una joven con diagnóstico de tartamudez, lo cual resultó evidente en su nivel de satisfacción con la influencia recibida, en la elevación del nivel de preparación de los profesores que interactúan con ella en el contexto universitario, y los alumnos del grupo, así como en el bienestar provocado a su familia con las orientaciones realizadas.

El gabinete logopédico se consolida como espacio por excelencia para la inclusión educativa desde la labor de los especialistas de Logopedia y su trabajo colaborativo con alumnos ayudantes, profesores y familias.

\section{Referencias}

Arguelles, A (2019). Intervención logopédica a través de la utilización de las TICs, para mejorar las habilidades articulatorias en niños de 3 a 7 años que presentan dislalias en el consultorio logopédico de la doctora Neyra Hernández Castro (Tesis de pregrado). Universidad Politécnica Salesiana. Quito.

Amaguaya, J. P. (2013). Intervención logopedia indirecta para los niños de 5 a 6 años con dislalia. Elaboración y aplicación de una guía didáctica para docentes y representantes (Proyecto Educativo). Universidad de Guayaquil. Facultad de Filosofía, Letras y Ciencias de la Educación.

Beiain, T. B. (2015). Tartamudez, una disfluencia con cuerpo y alma. Buenos Aires: Paidos.

Bonilla, M., Bimilla, V. \& Mosquera, L. (2014). Percepciones de las habilidades comunicativas, sociales cognitivas, psicoemocionales y psicomotrices de un grupo de adultos jóvenes con tartamudez de la ciudad de Santiago de Cali. Trabajo de grado I y II. Escuela de rehabilitación humana. Programa Académico de Fonoaudiología.

Booth, T. \& Ainscow, M. (2000). Índice de inclusión el aprendizaje y la participación en las escuelas (versión en castellano). Bristol: Center for Studies on Inclusive Education. 
Blood, G. \& Blood, I. (2004). Bullying in Adolescents Who Stutter: Communicative Competence and Self-Esteem. Contemporary Issues In Communication Science And Disorders, 31, 69-79. Recuperado de http://www.scirus.com

Castejón, L. A., \& España, Y. (2004). La colaboración logopeda-maestro: hacia un modelo inclusivo de intervención en las dificultades del lenguaje. Revista de Logopedia, Foniatría y Audiología, 24(2), 55-66.

Castejón, L., González, S., Núñez., J. C. \& González, A. (2008). La escuela inclusiva ante los alumnos tartamudos: actitudes y emociones en la relación educativa. Revista de Educación, 345, 281-299.

Chernousova, L. (2008). La estimulación de la comunicación verbal de los escolares con necesidades educativas especiales en el desarrollo general del lenguaje. (Tesis de doctorado). ISPH. Holguín.

Delgado, I., Navarro, Y., Vigoa, A., Castro, G. \& Martínez, A. (2016). Laboratorio logopédico para la atención a la disfonía desde la formación inicial y permanente. Ciencias Médicas de Pinar del Río, 20(6), 751-759.

Echeita, G. (2006). Educación para la inclusión o educación sin exclusiones. Madrid: Narcea.

Echeita, G. \& Sandoval, M. (2002). Educación inclusiva o educación sin exclusiones. Revista de Educación, 327, 31-48.

Figueredo, E. \& López, M. (1984). Logopedia I. La Habana: Pueblo y Educación.

Figueredo, E. \& López, M. (1986). Logopedia I. La Habana: Pueblo y Educación.

Fernández, G. (2008). Atención logopédica en la edad infantil. La Habana: Pueblo y Educación.

Gallego, J. L. (1999). Calidad en la intervención del lenguaje. Málaga: Aljibe.

Gallego, J. L. (2013). Los tratarnos del lenguaje en el niño. Estudio de caso. España: MAD, S. L.

Gallego, J. L. (2016). Las dificultades del lenguaje oral. Atención psicoeducativa en la escuela infantil. Granada, España: Técnica AVICAM.

Garaigordobil, M. \& Pérez, J. (2007). Autoconcepto, autoestima y síntomas psicopatológicos en personas con y sin disfemia: un análisis descriptivo y comparativo. International Journal of Psychology and Psychological Therapy,7(2),285-298.

Guitar, B. (1998). Stuttering: An integrated approach to its nature and treatment. Massachussets: Williams and Wilkins.

Hernández, T. B., Hernández, T. \& Rodríguez, B. (2019). Gabinete logopédico: espacio de extensión universitaria para la atención a niños con síndrome de Down. Edumecentro. Santa Clara, 11(3). Recuperado de http://scielo.sld.cu/scielo.php?script=sci_arttext\&pid=S207728742019000300006

Horruitiner, P. (2009). La Universidad Latinoamericana en la Época Actual: tendencias, retos y propuestas innovadoras. Sello editor Educación Cubana. Ministerio de Educación. Curso23. Recuperado http://www.cubaeduca.cu/media/www.cubaeduca.cu/medias/pdf/4754.pdf

de: 
Klompas, M.Y. \& Ross, E. (2004). Life experiences of people who stutter, and the perceived impact of stuttering on quality of life: personal accounts of South African individuals. Journal of Fluency Disorders, 29, 275-305.

Marchesi, A. (2004). Qué será de nosotros, los malos alumnos. Madrid: Alianza ensayo.

Miras, M. (1998). Aspectos afectivos y relacionales en la interacción educativa. En Psicología de la Instrucción (vol. 3) componentes contextuales y relacionales del aprendizaje escolar. Barcelona: EUB.

Morales, M. E. (2004). Alternativa metodológica de intervención logopédica para potenciar el desarrollo del lenguaje oral de los alumnos con retraso mental leve del grado preparatorio (Tesis presentada en opción al grado científico de Doctor en Ciencias Pedagógicas). Universidad de Ciencias Pedagógicas "Enrique José Varona”, La Habana, Cuba.

Moreno, J. (2017). Análisis y valoración de un programa de intervención logopédica como elemento de mejora de la práctica docente en la especialidad de audición y lenguaje. Un Modelo de investigación-acción en un IES de Ceuta (Tesis doctoral). Universidad de Granada. Facultad de Educación, Economía y Tecnología.

Monfort, M. (2005). Intervención logopédica en los trastornos de comprensión. Revista Neurología, 40(1),127- 130.

Noguera, K. (2008). La integración educativa en el niño de tres a cinco años de edad con déficit auditivo al grado preescolar de la escuela primaria (Tesis presentada en opción al grado científico de Doctor en Ciencias Pedagógicas). UCP "José de la Luz y Caballero", Holguín.

Pérez, E. (2002). Programa de ayuda logopédica para la prevención de la tartamudez (Tesis presentada en opción al grado científico de Doctor en Ciencias Pedagógicas). Instituto Superior Pedagógico "José de la Luz y Caballero". Facultad de Educación Infantil. Departamento de Educación Especial. Holguín.

Rodríguez, P. (2002). La tartamudez desde la perspectiva de los tartamudos. Caracas: Universidad Central de Venezuela. Facultad de Humanidades y Educación Comisión de Estudios de Postgrado. Área Psicología.

Tovar, (2016). La tartamudez: una revisión bibliográfica (Máster en patologías del lenguaje y del habla). Instituto Superior de estudios psicológicos. Chile.

UNESCO, (2017). La UNESCO Avanza. La Agenda 2030 para el Desarrollo Sostenible. París: Organización de las Naciones Unidas para la Educación, La Ciencia y la Cultura.

Valdés, A. I., Fernández. G., Perojo, D. A. (2017). Atención logopédica integral y comunitaria desde la formación inicial y permanente. Asociación de Pedagogos de Cuba. Capítulo Cuba de la Asociación de Educadores de América Latina y del Caribe (AELAC) VI intercambio entre educadores de Latinoamérica. Pinar del Río.

Ygual, J. F. \& Cervera (1999). La intervención logopédica en los trastornos de la adquisición del lenguaje. Revista de Neurología, 28(2), 109-18. 